\title{
MAXIMAL AND MINIMAL SOLUTIONS OF SECOND ORDER ELLIPTIC AND PARABOLIC EQUATIONS IN NON-DIVERGENCE FORM WITH MEASURABLE COEFFICIENTS
}

\author{
M. CRISTINA CERUTTI AND ANNA PIRO GRIMALDI
}

(Communicated by Tatiana Toro)

\begin{abstract}
In this paper we will prove that the supremum and infimum of good solutions of the Dirichlet problem for elliptic and parabolic equations in non divergence form with measurable coefficients, are good solutions to the same problem.
\end{abstract}

\section{INTRODUCTION}

Let $D \subset \mathbf{R}^{n}$ be a bounded domain with smooth boundary $\partial D$ and $I=(0, T)$ be an interval; then $Q$ will be the cylinder $Q=D \times I$ in $\mathbf{R}^{n+1}$. In this paper $L$ will be either an elliptic operator defined in $D$ or a parabolic operator defined in $Q$ and, to point out that proofs of the main results are basically the same in both cases, we will use a notation that will not distinguish (unless necessary) among them. More precisely let $x=\left(x_{1}, \ldots, x_{n}\right)$ and $t \in \mathbf{R}$; then we will use the same letter $z$ for the variable understanding that $z=x$ in the context of elliptic operators or $z=(x, t)$ in the context of parabolic operators. From know on we will also use $N$ meaning $N=n$ in the elliptic context and $N=n+1$ in the parabolic one. So e.g. we will write $z \in \mathbf{R}^{N}$. The operator $L$ will have either the form

$$
L=\sum_{i, j=1}^{n} a_{i j}(z) D_{i j}
$$

for $z \in D$ or the form

$$
L=\sum_{i, j=1}^{n} a_{i j}(z) D_{i j}-\partial_{t}
$$

for $z \in Q$. Here we use the notation $D_{i j}=\frac{\partial^{2}}{\partial x_{i} \partial x_{j}}$ In both cases $a_{i j}=a_{j i}$ are measurable functions such that

$$
\lambda|\xi|^{2} \leq \sum_{i, j=1}^{n} a_{i j}(z) \xi_{i} \xi_{j} \leq \Lambda|\xi|^{2}
$$

2010 Mathematics Subject Classification. 35J15, 35J25, 35K10,35K20 . 
for all $\xi \in \mathbf{R}^{n}$ and with $\lambda, \Lambda$ positive constants.

With the purpose of unifying even more the two contexts, when it is not necessary to distinguish we will use the same name $\Omega$ for the domain of the operators, understanding that $\Omega=D$ in the elliptic context and $\Omega=Q$ in the parabolic one. We will call $\mathcal{L}(\lambda, \Lambda, \Omega)$ the class of either elliptic or parabolic operators defined above.

Finally let the parabolic boundary of $Q$ be $\partial_{p} Q=\partial_{b} Q \cup \partial_{x} Q$, where $\partial_{b} Q=$ $D \times\{0\}$ and $\partial_{x} Q=\partial D \times \bar{I}$. Accordingly, we will use the notation $\partial_{d} \Omega$ to mean $\partial D$ in the elliptic context and to mean $\partial_{p} Q$ in the parabolic one.

For $g$ continuous on $\partial_{d} \Omega$ and $f \in L^{p}$ consider the following Dirichlet problem (D.P):

$$
\left\{\begin{array}{ccc}
L u=f & \text { on } & \Omega \\
u=g & \text { on } & \partial_{d} \Omega
\end{array}\right.
$$

If the coefficients $a_{i j}$ are at least continuous functions in $\bar{\Omega}$ the problem has a unique solution $u \in W_{p}^{2}\left(\Omega^{\prime}\right)$ if $L$ is elliptic or $u \in W_{p}^{2,1}\left(\Omega^{\prime}\right), p>1$ if $L$ is parabolic, for any $\Omega^{\prime} \subset \bar{\Omega}^{\prime} \subset \Omega$.

For discontinuous $a_{i j}$ it has been shown that Sobolev spaces are not suitable for the solvability of the above D.P., whereas counterexamples showed that either existence or uniqueness may fail, and such solutions may not satisfy basic properties, such as the maximum principle.

Therefore in several papers in the '90's (see e.g. [3],[8], [18]) a different notion of solution (good or weak solution) has been introduced. For sake of completeness we recall it in the following

Definition 1.1. A function $u(z) \in C(\bar{\Omega})$ is a good solution to problem (1.4) with $p=n$ in the elliptic case and $p=n+1$ in the parabolic, if

- there exist a sequence of operators $L^{k} \in \mathcal{L}(\lambda, \Lambda, \Omega)$ with coefficients $a_{i j}^{k} \in$ $\mathcal{C}(\bar{\Omega})$ such that $a_{i j}^{k} \rightarrow a_{i j}$ a.e. in $\Omega($ as $k \rightarrow \infty, i, j=1, \ldots, n)$. We will say $L^{K} \rightarrow L$.

- there exists a sequence of smooth functions $u^{k}$, solutions of the D.P.'s for the operators $L^{k}$ (defined respectively either as in (1.1) or as in (1.2) with coefficients $a_{i j}^{k}$ ), i.e.

$$
\left\{\begin{array}{ccc}
L^{k} u^{k}=f & \text { in } & \Omega \\
u^{k}=g & \text { on } & \partial_{d} \Omega
\end{array}\right.
$$

and such that $u_{k} \rightarrow u$ uniformly in $\bar{\Omega}$.

Recall that Krylov-Safonov uniform Hölder estimates hold for the $u^{k}$ 's independently of the regularity of the coefficients. This implies that the functions $u^{k}$ are uniformly bounded and equicontinuous in $\Omega$ and by Ascoli-Arzela's theorem, there exists a convergent subsequence. Therefore good solutions always exist.

A result by N. Nadirashviili for elliptic operator (see [13]), states that uniqueness for good solutions may fail if $n \geq 3$. He constructs two sequences of operators with smooth coefficients $a_{i j}^{0, k}$ and $a_{i j}^{1, k}$ which satisfy the ellipticity condition with the same constants and converge to the same $a_{i j}$ a.e., as $k \rightarrow \infty$, in the unit ball $B_{1} \subset R^{3}$, while the corresponding sequences of solutions converge to two different functions 
$u^{0}$ and $u^{1}$. Because of the probabilistic nature of Nadirashvili's counterexample, it is reasonable to think that it can be slightly modified to provide a counterexample also in the parabolic setting.

In this paper we show that, if uniqueness does not hold, the supremum and the infimum of good solutions are still good solutions. The result is not new for the elliptic case, since it is known to hold for viscosity solutions and Jensen in [7] shows that good solutions in this case coincide with viscosity solutions. Our result, though, besides being new for parabolic equations, provides a unifying proof for both cases and a direct method also for elliptic case.

We hope that this result could be helpful in improving the known results about uniqueness. In fact Nadirashvili's example did not settle completely the matter, since the set of discontinuities of his operators is "very large". Several results for uniqueness (see i.g. [3] and [18] for elliptic equations and [4] for parabolic equations) have been proved that when the set of discontinuities is not too bad, but many problems still remain open such as that of discontinuities along a general line segment or a general hyperplane.

The main result is proved in section 4 and a main role in the proof is played by the parabolic version of Pucci's extremal operators, whose definitions and main properties are recalled in section 2.

We wish to thank professor Paolo Manselli for very helpful suggestions and discussions.

\section{Definitions AND PRELIMINARY RESUlts}

A main role in the proofs is played by Pucci's extremal operators (see [14]) that we recall here. We will as well introduce the parabolic version of these operators.

For a symmetric matrix $M \in \mathcal{S}$ (space of real $n \times n$ symmetric matrices), with eigenvalues $e_{1}, \ldots, e_{n}$, define

and

$$
\mathcal{M}^{-}(M, \lambda, \Lambda)=\mathcal{M}^{-}(M)=\lambda \sum_{e_{i}>0} e_{i}+\Lambda \sum_{e_{i}<0} e_{i}
$$

$$
\mathcal{M}^{+}(M, \lambda, \Lambda)=\mathcal{M}^{+}(M)=\Lambda \sum_{e_{i}>0} e_{i}+\lambda \sum_{e_{i}<0} e_{i} .
$$

Let $\mathcal{A}_{\lambda, \Lambda}$ be the set of symmetric matrices with eigenvalues in $[\lambda, \Lambda]$. Define the linear functional $L_{A}$ on $\mathcal{S}$ by

$$
L_{A} M=\operatorname{tr}(A M)
$$

It is well known (see [2]) that

$$
\mathcal{M}^{-}(M)=\inf _{A \in \mathcal{A}} L_{A} M \quad \text { and } \quad \mathcal{M}^{+}(M)=\sup _{A \in \mathcal{A}} L_{A} M .
$$

Elliptic Pucci's extremal operators are defined as:

$$
L^{+} u=\mathcal{M}^{+}\left(D^{2} u\right) \quad \text { and } \quad L^{-} u=\mathcal{M}^{-}\left(D^{2} u\right)
$$

while parabolic Pucci's extremal operators as:

$$
L^{+} u=\mathcal{M}^{+}\left(D^{2} u\right)-u_{t} \quad \text { and } \quad L^{-} u=\mathcal{M}^{-}\left(D^{2} u\right)-u_{t} .
$$


We'll also need Hölder and Sobolev spaces and their parabolic counterpart that we'll recall below.

Given $0<\alpha \leq 1$ the subspace of $C(\Omega)$ of $\alpha$-Hölder continuous functions is defined as the space of functions $u$ such that the norm

$$
\|u\|_{\mathcal{C}^{\alpha}(\Omega)}=\sup _{z \in \Omega}|u(z)|+\sup _{z_{1}, z_{2} \in \Omega} \frac{\left|u\left(z_{1}\right)-u\left(z_{2}\right)\right|}{d\left(z_{1}, z_{2}\right)^{\alpha}}
$$

is finite, with $d$ the usual Euclidean distance.

For the parabolic context, we recall a definition of parabolic distance between $z_{1}=\left(x_{1}, t_{1}\right), z_{2}=\left(x_{2}, t_{2}\right)$ :

$$
d\left(z_{1}, z_{2}\right)=\left\{\begin{array}{cc}
\max \left\{\left|x_{1}-x_{2}\right|,\left|t_{1}-t_{2}\right|^{1 / 2}\right\} & t_{1} \leq t_{2} \\
\infty & t_{1}>t_{2}
\end{array}\right.
$$

Now given $0<\alpha \leq 1$, the subspace of $C(\Omega)$ of parabolic $\alpha$-Hölder continuous functions is defined as the space of functions $u$ that satisfy (2.1) with $d$ the parabolic distance.

As before, throughout the paper, the space $C^{\alpha}(\Omega)$ will mean either the space of classical or that of parabolic Hölder continuous functions.

Moreover in what follows, let $W(\Omega)$ be the Sobolev space $W_{n}^{2}(\Omega)$ in the elliptic context and $W_{n+1}^{2,1}(\Omega)$ for the parabolic one.

The space $W_{\text {loc }}(\Omega)$ is the space of functions $f$ defined on $\Omega$ such that $f \in W\left(\Omega^{\prime}\right)$ for every $\Omega^{\prime} \subset \bar{\Omega}^{\prime} \subset \Omega$.

Finally $B_{R}\left(z_{0}\right)=\left\{z \in \mathbf{R}^{N} \mid d\left(z, z_{0}\right)<R\right\}$ will be the Euclidean ball of radius $R$ in the elliptic context and a parabolic cylinder otherwise.

We will need the following results :

Theorem 2.1. (ABP/ABPKT-Maximum principle)(see [1] and [15] for elliptic operators and [9] and [19] for parabolic; a more general version for both cases in $[5])$. Let $L$ be either the elliptic operator (1.1) or the parabolic opertor (1.2), $u \in$ $W_{\text {loc }}(\Omega) \cap \mathcal{C}(\bar{\Omega})$, then there exists $C=C(\lambda, \Lambda, N, \Omega)$ such that

$$
\sup _{\Omega}|u| \leq \sup _{\partial_{d} \Omega}|u|+C\|L u\|_{L^{N}(\Omega)} .
$$

Theorem 2.2. (Hölder estimate, see i.e. [16] and [6] for elliptic, [10] and [12] for parabolic) Let $L$ be either an elliptic or a parabolic operator with smooth coefficients, $u \in W_{\text {loc }}(\Omega), B_{R_{0}}(z) \subset \Omega$, then there exist constants $K\left(N, \lambda, \Lambda, R_{0}\right)$ and $\alpha(N, \lambda, \Lambda)$, such that for $R<R_{0}$

$$
\|u\|_{\mathcal{C}^{\alpha}\left(B_{R}\right)} \leq K\left(\|u\|_{\infty}+\|L u\|_{N}\right)
$$

Moreover, if $u \in W(\Omega)$ and $u=0$ on $\partial_{d} \Omega$, there exists $C_{2}=C_{2}(\lambda, \Lambda, N, \Omega)$ such that

$$
\|u\|_{\mathcal{C}^{\alpha}(\bar{\Omega})} \leq S_{2}\left(\|u\|_{\infty}+\|L u\|_{N}\right)
$$

Theorem 2.3. (see [6] and [12]) Let $\mathcal{L}$ be a finite or countable family of operators $L^{k}$ with coefficients $C^{1}(\bar{\Omega})$. Define

$$
F_{\mathcal{L}}(u)=\sup _{L \in \mathcal{L}} L u .
$$


Then there exists $\beta=\beta(N, \lambda, \Lambda) \in(0,1)$ such that, if $f \in \mathcal{C}^{1}(\Omega), g \in \mathcal{C}\left(\partial_{d} \Omega\right)$, the problem

$$
\left\{\begin{array}{ccc}
F_{\mathcal{L}}(u)=f & \text { in } & \Omega \\
u=g & \text { on } & \partial_{d} \Omega
\end{array}\right.
$$

has a unique solution $u \in \mathcal{C}_{\text {loc }}^{2, \beta}(\Omega) \cap C(\bar{\Omega})$.

\section{Properties of Good and Classical solutions}

Let $\mu>0, \Omega_{\mu}=\left\{z \in \Omega: \operatorname{dist}\left(z, \partial_{d} \Omega\right)>\mu\right\} \subset \Omega$.

Lemma 3.1. Let $L \in \mathcal{L}(\lambda, \Lambda, \Omega)$ and $a_{i j} \in C(\bar{\Omega}), u \in W(\Omega) \cap C(\bar{\Omega})$. If $L u=f$, $\left.u\right|_{\partial_{d} \Omega}=g$ and $f \in L^{N}(\Omega), g \in C\left(\partial_{d} \Omega\right)$, then there exists $\sigma_{\mu}>0, \sigma_{\mu} \rightarrow 0$ as $\mu \rightarrow 0$, satisfying:

$$
\sup _{z_{\mu} \in \partial_{d} \Omega_{\mu}} \inf _{\xi \in \partial_{d} \Omega}\left|g(\xi)-u\left(z_{\mu}\right)\right| \leq \sigma_{\mu}
$$

The number $\sigma_{\mu}=\sigma\left(\lambda, \Lambda, \Omega, \mu, N,\|f\|_{L^{N}(\Omega)}, g\right)$.

Proof. Assume for the moment $f=0$ a.e. Let $\mathcal{L}^{*}=\{L \in \mathcal{L}: L$ has rational constant coefficients in $\Omega\}$ and consider Pucci's maximal and minimal operators:

$$
L^{+} u=F_{\mathcal{L}^{*}}(u) \quad \text { and } \quad L^{-} u=-F_{\mathcal{L} *}(-u) .
$$

By Theorem 2.3 there exist $u_{\mu}^{+}, u_{\mu}^{-} \in C^{2, \beta}(\Omega) \cap C(\bar{\Omega})$ satisfying:

$$
\left\{\begin{array}{ccc}
L^{+}\left(u_{\mu}^{+}\right)=0 & \text { in } & \Omega \\
u_{\mu}^{+}=g & \text { on } & \partial_{d} \Omega
\end{array}\right.
$$

and

$$
\left\{\begin{array}{ccc}
L^{-}\left(u_{\mu}^{-}\right)=0 & \text { in } & \Omega \\
u_{\mu}^{-}=g & \text { on } & \partial_{d} \Omega
\end{array}\right.
$$

As $u_{\mu}^{+}$and $u_{\mu}^{-}$are continuous functions, then:

$$
\begin{gathered}
\left.\sigma_{\mu}^{-}=\sup \mid g(\xi)-u_{\mu}^{-}\left(z_{\mu}\right)\right) \mid \rightarrow 0 \text { as } \mu \rightarrow 0 \\
\sigma_{\mu}^{+}=\sup \left|u_{\mu}^{+}\left(z_{\mu}\right)-g(\xi)\right| \rightarrow 0 \text { as } \mu \rightarrow 0
\end{gathered}
$$

where $z_{\mu} \in \partial_{d} \Omega_{\mu}$ and $\xi \in \partial_{d} \Omega$

As $L u_{\mu}^{+} \leq 0$ and analogously $L u_{\mu}^{-} \geq 0$ we have $L\left(u-u_{\mu}^{+}\right) \geq 0$ and $L\left(u_{\mu}^{-}-u\right) \geq 0$ in $\Omega$. By the Maximum Principle:

$$
\begin{aligned}
& \sup _{\Omega}\left(u-u_{\mu}^{+}\right) \leq 0 \\
& \sup _{\Omega}\left(u_{\mu}^{-}-u\right) \leq 0 .
\end{aligned}
$$

We can write:

$$
\begin{aligned}
& u\left(z_{\mu}\right)-g(\xi) \leq \sup \left(u-u_{\mu}^{+}\right)+\sigma_{\mu}^{+} \leq \sigma_{\mu}^{+} \\
& g(\xi)-u\left(z_{\mu}\right) \leq \sigma_{\mu}^{+}+\sup \left(u_{\mu}^{-}-u\right) \leq \sigma_{\mu}^{-},
\end{aligned}
$$

i.e.

$$
\left|u\left(z_{\mu}\right)-g(\xi)\right| \leq \max \left(\sigma_{\mu}^{+}, \sigma_{\mu}^{-}\right) .
$$

The thesis follows, if $f$ vanishes a.e. 
If $f$ is non-identically zero, we can write $u=u_{1}+u_{2}$, where $u_{1} \in W(\Omega), L u_{1}=f$ in $\Omega$ and $u_{1}=0$ on the boundary $\partial_{d} \Omega, u_{2} \in W(\Omega), L u_{2}=0$ in $\Omega$ and $u_{2}=g$ on the boundary. Then $u_{1}$ satisfies Hölder estimates of Theorem 1, namely

$$
\sup _{z_{\mu} \in \partial_{d} \Omega_{\mu}}\left|u\left(z_{\mu}\right)\right| \leq \mu^{\alpha} K(1+C)\|f\|_{L^{N}(\Omega)}
$$

The thesis follows with $\sigma_{\mu}=\max \left(\sigma_{\mu}^{+}, \sigma_{\mu}^{-}, \mu^{\alpha} K(1+C)\|f\|_{L^{N}(\Omega)}\right)$.

As a consequence, an equicontinuity result follows:

$\forall z_{1}, z_{2} \in \bar{\Omega}$ such that $d\left(z_{1}, z_{2}\right) \leq \delta$ and for any $u \in W(\Omega) \cap C(\bar{\Omega})$ with $\|L u\|_{L^{N}} \leq$ $F$ and $u=g$ on the boundary; there exists $\omega(\delta) \rightarrow 0$ as $\delta \rightarrow 0$, such that:

$$
u\left(z_{1}\right)-u\left(z_{2}\right) \mid \leq \omega(\delta)
$$

Remark 3.2. If the D.P.(1.4) has a strong solution $u \in W(\Omega)$, then $u$ is also the unique good solution to this problem.

Proof. Let $L^{k} \in \mathcal{L}(\lambda, \Lambda, \Omega), k=1,2, \ldots$, with coefficients $a_{i j}^{k}$ that are smooth in $\bar{\Omega}$, $L^{k} \rightarrow L$ as $k \rightarrow \infty$ and let $\left\{u^{k}\right\}$ be a sequence of solutions to the problems (1.5). Then

$$
\left\{\begin{array}{ccc}
L^{k}\left(u^{k}-u\right)=\left(L-L^{k}\right) u & \text { on } & \Omega \\
u^{k}-u=0 & \text { on } & \partial_{d} \Omega
\end{array}\right.
$$

Since $u^{k}-u \in W$, by the A.B.P.K.T Theorem 2.1, we have

$$
\sup _{\Omega}\left|u^{k}-u\right| \leq K\left\|\left(L-L^{k}\right) u\right\|_{L^{N}(\Omega)}=K\left(\int_{\Omega}\left|\sum_{i, j}\left(a_{i j}-a_{i j}^{k}\right) D_{i, j} u\right|^{N} d x d t\right)^{1 / N}
$$

with constant $\mathrm{K}$ independent of $k$. Since the argument in the integral converges to 0 a.e. in $\Omega$ as $k \rightarrow \infty$, we have

$$
\lim _{k \rightarrow \infty} \sup _{\Omega}\left|u^{k}-u\right|=0
$$

This means that $u(z)$ is the only good solution to the problem (1.4).

Remark 3.3. Let $L, L^{k} \in \mathcal{L}, f, f^{k} \in L^{N}(\Omega) g \in C\left(\partial_{d} \Omega\right), L^{k} \rightarrow L$ and $f^{k} \rightarrow f$ a.e. (meaning the coefficients $a_{i j}^{k}$ of $L^{k}$ converge a.e. to $a_{i j}$ of $L$ as in Definition 1.1) in $\Omega$ and let $u^{k} \in C(\bar{\Omega})$ be good solutions to

$$
\left\{\begin{array}{ccc}
L^{k} u^{k}=f^{k} & \text { on } & \Omega \\
u^{k}=g & \text { on } & \partial_{d} \Omega
\end{array}\right.
$$

then, there exists a subsequence of $u^{k}$ uniformly convergent in $\bar{\Omega}$ to a function $u \in C(\bar{\Omega})$ good solution to (1.4).

Proof. The operators $\bar{L}^{k}$ can be chosen with coefficients in $C^{1}(\bar{\Omega})$. As $\left\{\left\|f_{n}\right\|_{L^{N}(\Omega}\right\}$ is a bounded sequesnce, by Theorem 2.1, the equicontinuity (3.4) of the $u_{k}$ 's and Ascoli-Arzelá theorem, a subsequence of $\left\{u_{k}\right\}$ (still named $\left\{u_{k}\right\}$ ) converges uniformly in $\bar{\Omega}$ to a function $u \in \mathcal{C}\left(\bar{\Omega}\right.$. Since $u^{k}$ 's are good solutions to the problem (3.5) there exist $\bar{L}^{k}$ with $\bar{a}_{i j}^{k} \in C^{1}(\bar{\Omega})$, satisfying 


$$
\sum_{i, j}\left\|a_{i j}^{k}-\bar{a}_{i j}^{k}\right\|_{L(\Omega)} \leq 1 / k
$$

and functions $v^{k} \in W(\Omega) \cap C(\bar{\Omega})$, satisfying

$$
\left\{\begin{array}{ccc}
\bar{L}^{k} v^{k}=f^{k} & \text { on } & \Omega \\
v^{k}=g & \text { on } & \partial_{d} \Omega
\end{array}\right.
$$

and $\sup _{\Omega}\left|v^{k}-u^{k}\right| \leq 1 / k ;$ as $\bar{L}^{k} \rightarrow L$ and (a subsequence of) $v^{k} \rightarrow u$ uniformly in $\bar{\Omega}$. Consider now functions $w^{k} \in W(\Omega) \cap C(\bar{\Omega})$ such that

$$
\begin{gathered}
\left\{\begin{array}{ccc}
\bar{L}^{k} w^{k}=f & \text { on } & \Omega \\
w^{k}=g & \text { on } & \partial_{d} \Omega
\end{array}\right. \\
\left\{\begin{array}{ccc}
\bar{L}^{k}\left(w^{k}-v^{k}\right)=f-f^{k} & \text { on } & \Omega \\
w^{k}-v^{k}=0 & \text { on } & \partial_{d} \Omega ;
\end{array}\right.
\end{gathered}
$$

by A.B.P.K.T Theorem 2.1 we have

$$
\sup _{\Omega}\left|w^{k}-v^{k}\right| \leq K\left\|f-f^{k}\right\|_{L^{N}}
$$

As a consequence $w^{k} \rightarrow u$ uniformly in $\bar{\Omega}$ and $u$ is a good solution to problem (1.4) (with approximating operators $\bar{L}^{k}$ ).

From Remark 3.2 and Remark 3.3 if $u \in C(\bar{\Omega})$ is a good solution to problem (1.4) with $f \in \mathcal{C}^{1}$ then $u$ is a good solution to the same problem with $a_{i j}^{k} \in C^{1}(\bar{\Omega})$ and functions $u^{k} \in C^{2, \alpha}(\Omega) \cup C(\bar{\Omega}), 0<\alpha<1$.

\section{MAIN RESUlt}

Lemma 4.1. Let $L \in \mathcal{L}, f \in C^{1}(\bar{\Omega})$. Let $u_{1}, u_{2}, \ldots, u_{S}$ be good solutions to (D.P.) (1.4) with $f \in \mathcal{C}^{1}$. Then, there exists $v$, good solution to $L v=f$ in $\Omega,\left.v\right|_{\partial_{d} \Omega}=g$, such that

$$
v(z) \geq \max \left\{u_{1}(z), \ldots, u_{S}(z)\right\}
$$

in $\bar{\Omega}$.

Proof. We may assume, by previous observations, that $u_{l}(l=1,2, \ldots S)$ is a good solution to D.P. (1.4) with $L_{l}^{k}$ in the definition of good solution with coefficients in $C^{1}(\bar{\Omega})$; the corresponding $u_{l}^{k} \in C^{2, \alpha}(\Omega) \cap C(\bar{\Omega}), 0<\alpha<1$. Moreover, as recalled above, if

$$
F^{k}(\omega)=\max \left(L_{1}^{k} \omega, L_{2}^{k} \omega, \ldots, L_{S}^{k} \omega\right)
$$

the Dirichlet problem

$$
\left\{\begin{array}{ccc}
F^{k}\left(v^{k}\right)=f & \text { in } & \Omega \\
v^{k}=g & \text { on } & \partial_{d} \Omega
\end{array}\right.
$$

has a unique solution $v^{k} \in C^{2, \beta}(\Omega) \cup C(\bar{\Omega}), 0<\beta<1$. 
Notice that the equation $F^{k}\left(v^{k}\right)=f$ can also be written as $L^{k} v^{k}=f$, where $L^{k} v^{k}(z)=L_{l(z)}^{k} v^{k}(z)$ and the operators $L^{k} \in \mathcal{L}$ and have measurable coefficients. Moreover $L^{k} \rightarrow L$ a.e. in $\Omega$. By A.B.P. or A.B.P.K.T Theorem 2.1 and Hölder estimate Theorem 2.2 , the sequence $v^{k}$ converges uniformly in $\Omega$ to $v$ good solution to (1.4).

As $L^{k}{ }_{l} v^{k} \leq f=L^{k}{ }_{l} u_{l}^{k}$ and $u_{l}^{k}=v^{k}=g$ on the boundary, by the Maximum Principle: $v^{k} \geq u_{l}^{k}$. As $k \rightarrow \infty$ (4.1) follows.

Here is our main result:

Theorem 4.2. Let $L, f, g$ be as in Lemma 4.1, and let $\mathcal{H}$ be the family of all good solutions to the Dirichlet Problem: Lu=f in $\Omega$ and $u=g$ on the boundary $\partial_{d} \Omega$. Then

$$
\begin{aligned}
U_{0}(z) & =\sup \{u(z): u \in \mathcal{H}\} \\
V_{0}(z) & =\inf \{u(z): u \in \mathcal{H}\}
\end{aligned}
$$

for $z \in \Omega$ are good solutions to the same problem.

Proof. Assume that $\mathcal{H}$ has more than one element (see [13]). Let us show that $U_{0}$ exists and is continuous in $\bar{\Omega}$.

By ABPKT Theorem 2.1, $U_{0}$ is bounded in $\Omega ; \forall z_{0}, z_{1} \in \bar{\Omega}$ let $u^{k} \in \mathcal{H}$ sequence such that $u^{k}\left(z_{0}\right) \rightarrow U_{0}\left(z_{0}\right)$ and by the equicontinuity result, we have

$$
u^{k}\left(z_{0}\right)-u^{k}\left(z_{1}\right) \leq \omega\left(d\left(z_{0}, z_{1}\right)\right)
$$

where $\omega=\omega(\lambda, \Lambda, \Omega, N, g)$.

Since $U_{0}\left(z_{1}\right) \geq u^{k}\left(z_{1}\right)$, we have

$U_{0}\left(z_{0}\right)-U_{0}\left(z_{1}\right) \leq U_{0}\left(z_{0}\right)-u^{k}\left(z_{0}\right)+u^{k}\left(z_{0}\right)-u^{k}\left(z_{1}\right) \leq U_{0}\left(z_{0}\right)-u^{k}\left(z_{0}\right)+\omega\left(d\left(z_{0}, z_{1}\right)\right)$ so, as $k \rightarrow \infty, U_{0}$ is continuous in $\bar{\Omega}$ with the same modulus of continuity $\omega$ as $u_{k}$. Assume for the moment $f \in \mathcal{C}^{1}(\bar{\Omega})$. For every $\epsilon>0$, there exist $z_{l} \in \bar{\Omega}, l=1,2, \ldots S$, so that $\bar{\Omega}$ is covered by sets $B_{\epsilon}\left(z_{l}\right)$ (defined in Section2.) in which osc $U_{0} \leq \epsilon$ and osc $u \leq \epsilon$ for every $u \in \mathcal{H}$.

Let now $u_{1}, u_{2}, \ldots, u_{S} \in \mathcal{H}$ so that

$$
\left|u_{l}\left(z_{l}\right)-U_{0}\left(z_{l}\right)\right|<\epsilon .
$$

By Lemma 4.1, there exists $v_{\epsilon} \in \mathcal{H}$, such that $v_{\epsilon} \geq u_{l}$ in $\bar{\Omega}$ and $v_{\epsilon} \leq U_{0}$ in $\bar{\Omega}$. Moreover $U_{0}-\epsilon \leq u_{l}\left(x_{l}\right) \leq v_{\epsilon}\left(x_{l}\right)$. Now choose $z \in \bar{\Omega}$ in one of the $B_{\epsilon}\left(z_{l}\right)$, thus:

$$
\begin{gathered}
0 \leq U_{0}(z)-v_{\epsilon}(z)= \\
{\left[U_{0}(z)-U_{0}\left(z_{l}\right)\right]+\left[U_{0}\left(z_{l}\right)-u_{l}\left(z_{l}\right)\right]+\left[u_{l}\left(z_{l}\right)-v_{\epsilon}\left(z_{l}\right)\right]+\left[v_{\epsilon}\left(z_{l}\right)-v_{\epsilon}(z)\right]=} \\
A+B+C+D .
\end{gathered}
$$

The terms $A$ and $D$ are $\leq \epsilon$ by the oscillation property in $B_{\epsilon}\left(z_{l}\right)$, term $B$ is $\leq \epsilon$ by (4.3), and term $\mathrm{C}$ is $\leq 0$. Then

$$
0 \leq U_{0}(z)-v_{\epsilon}(z) \leq 3 \epsilon
$$

Therefore the family $v_{\epsilon} \in \mathcal{H}$ converges uniformly to $U_{0}$ in $\bar{\Omega}$, as $\epsilon \rightarrow 0$ and $U_{0}$ is a good solution to $L u=f \in C^{1}(\bar{\Omega}), u=g$ on the boundary $\partial_{d} \Omega$.

For $f \in L^{N}$, let $f_{\nu} \in \mathcal{C}^{1}(\bar{\Omega}), f_{n} u \rightarrow f$ as $\nu \rightarrow \infty, \mathcal{H}_{\nu}$ the family of good solutions to $L u=f_{\nu}$ in $\Omega, f=g$ on the boundary $\partial_{d} \Omega$. Then $U_{\nu}^{0}=\sup \mathcal{H}_{\nu}$ are good solutions to the same problems, since the theorem is proved for $f_{\nu} \in \mathcal{C}^{1}(\bar{\Omega})$. By Remark 3.3 
up to a subsequence $u_{n} u$ converges uniformly to a function $w_{0}$ good dolution to to $L u=f$ in $\Omega, f=g$.

In order to show that $w_{0}=\sup \mathcal{H}$ let $u \in \mathcal{H}$ and $u_{\nu} \in \mathcal{H}_{\nu}$ with same approximating sequence $L^{k}$ in the definition fo good solution (Definition 1.1). Now $u_{\nu}^{0} \geq u_{\nu}$ implies $w_{0} \geq u$ and since $w_{0} \in \mathcal{H}$ we have proved the theorem.

The following result has been proved by Krylov in [8] for generalized Green's functions in the elliptic case.

Corollary 1. Let $L \in \mathcal{L}(\lambda, \Lambda, \Omega), f \in L^{N}(\Omega), g \in \mathcal{C}\left(\partial_{d} \Omega\right)$. Let $\mathcal{H}, U_{0}$ and $V_{0}$ be as in Theorem 4.2 and assume $U_{0} \neq V_{0}$. Then for every $\theta \in(0,1)$, we have that $t U_{0}(z)+(1-\theta) V_{0}(z) \in \mathcal{H}$.

Proof. We'll prove the result for $f \in \mathcal{C}^{1}(\bar{\Omega})$ as the result for general $f$ follows as in the proof of Theorem 4.2 .

Being $U_{0}$ and $V_{0}$ good solutions by previous theorem, let respectively $L_{k}^{(1)}, u_{k}^{(1)}$ and $L_{k}^{(2)}, u_{k}^{(2)}$ be approximating operators and funcions that appear in the definition of good solution.

Let $u_{k}^{+}, u_{k}^{-} \in \mathcal{C}^{2, \alpha}(\Omega) \cap \mathcal{C}(\bar{\Omega})$ be the solutions to the Dirichlet problems:

$$
\begin{aligned}
& \sup \left(L_{k}^{(1)} u_{k}^{+}, L_{k}^{(2)} u_{k}^{+}\right)=f \text { in }\left.\Omega \quad u_{k}^{+}\right|_{\partial_{d} \Omega}=g \\
& \inf \left(L_{k}^{(1)} u_{k}^{-}, L_{k}^{(2)} u_{k}^{-}\right)=f \text { in }\left.\Omega \quad u_{k}^{-}\right|_{\partial_{d} \Omega}=g
\end{aligned}
$$

There exist $L_{k}^{+}, L_{k}^{-} \in \mathcal{L}(\lambda, \Lambda, \Omega)$ such that

$$
L_{k}^{+} u_{k}^{+}=f, \quad L_{k}^{-} u_{k}^{-}=f \quad \text { in } \Omega
$$

As $L_{k}^{(1)} u_{k}^{+} \leq f, L_{k}^{(2)} u_{k}^{+} \leq f, L_{k}^{(1)} u_{k}^{-} \geq f, L_{k}^{(2)} u_{k}^{-} \geq f$ and on each point of $\Omega L_{k}^{+}$, $L_{k}^{-}$equal either $L_{k}^{(1)}$ or $L_{k}^{(2)}$ we get that

$$
L_{k}^{+} u_{k}^{-} \geq f, \quad L_{k}^{-} u_{k}^{+} \leq f
$$

Let $t \in(0,1), w_{n}=t u_{k}^{+}+(1-t) u_{k}^{-} \in \mathcal{C}^{2, \alpha}(\Omega) \cap \mathcal{C}(\bar{\Omega})$; the $w_{k}$ 's satisfy

$$
L_{k}^{+} w_{k}=t f+(1-t) L_{k}^{+} u_{k}^{-} \geq f, \quad L_{k}^{-} w_{k}=t f+(1-t) L_{k}^{-} u_{k}^{+} \leq f .
$$

Now let's define $L_{k}=\theta_{k} L_{k}^{+}+\left(1-\theta_{n}\right) L_{k}^{-}$, where

$$
\theta_{k}=\left\{\begin{array}{ll}
1 & \text { if } \\
\frac{f-L_{k}^{-} w_{k}}{L_{k}^{+} w_{k}-L_{k}^{-} w_{k}} & \text { otherwise }
\end{array} L_{k}^{+} w_{k}=L_{k}^{-} w_{k}=f\right.
$$

$\theta_{k}$ is a measurable function, $\theta_{k} \in[0,1], L_{k} \in \mathcal{L}(\lambda, \Lambda, \Omega)$ and $L_{k} w_{k}=f$ in $\Omega$. Observing that $L_{k}^{+}, L_{k}^{-}, L_{k} \rightarrow L$ as $k \rightarrow \infty$, Remarks 3.2 and 3.3 imply that there exist susequences still named $\left\{u_{k}^{+}\right\},\left\{u_{k}^{-}\right\}$that converge uniformly to elements of $\mathcal{H}$. By the maximum principle $u_{k}^{+} \geq u_{k}^{(1)}$ and $u_{k}^{-} \leq u_{k}^{(2)}$ and therefore $u_{k}^{+} \rightarrow U_{0}$ and $u_{k}^{-} \rightarrow V_{0}$ and $w_{k} \rightarrow t U_{0}+(1-t) V_{0}$ uniformly in $\Omega$. This proves that the latest is in $\mathcal{H}$. 


\section{REFERENCES}

[1] A.D.Aleksandrov Uniqueness conditions and bounds for the solution of the Dirichlet problem. Vestnik Leningrad. Univ. Ser. Mat. Meh. Astronom. 18 no. 3, (1963) 5-29 (Russian) ; English tranl. in Amer. Math. Soc. Transl. (2) 68, (1968) 89-119.

[2] L.A.CAffarelli And X.CABré Fully nonlinear elliptic equations. AMS Colloquium Publications, vol 43 (1995).

[3] M.C.Cerutti, L.Escauriaza and E.B.Fabes, Uniqueness in the Dirichlet problem for elliptic equations with discontinuous coefficients, Ann.Mat.Pura Appl. (4) 163 (1993), 161-180.

[4] M.C.Cerutti and A.Piro Grimaldi, Uniqueness for second order parabolic equations with discontinuous coefficients, Ann.Mat.Pura Appl. (4) 186 (2007), 147-155.

[5] E.B. Fabes and D.W. Stroock, The $L^{p}$ integrability of Green's functions and fundamental solutions for elliptic and parabolic equations, Duke Math. J. 51 (1984), 997-1016.

[6] D. Gilbarg and N.S. Trudinger Elliptic Partial Differential Equations of Second Order, Springer-Verlag, Berlin/Heidelberg/New York 1977.

[7] R.R. Jensen, Uniformly elliptic PDEs with bounded, measurable coefficients., J. Fourier Anal. Appl. 2 (1996), no. 3, 237-259.

[8] N.V.Krylov, On one-point weak uniqueness for elliptic equations., Comm. Partial Differential Equations 17 (1992), no. 11-12, 1759-1784.

[9] N.V. Krylov, Sequences of convex functions and estimates ofthe maximum of the solutions of a parabolic equation, (Russian) Sibirsk. math. Zh.17 (1976), 290-303; English translation in Siberian Math. J. 17, (1976), 226-236.

[10] N.V.Krylov and M.V.Safonov, A property of the solutions of parabolic equations with measurable coefficients, (Russian) Izv. Akad. Nauk SSSR Ser. Mat. 44 (1980) n.1, 161-175; English translation in Math.USSR.Izv. 16, (1981), 155-164.

[11] E.M.Landis Second order equations of elliptic and parabolic, type, "Nauka", Moscow, 1971 (in Russian); English transl.: American Mathematical Soc.,Tranls.Math.Monographs, vol 171, Providence, RI. 1997.

[12] G.M.Lieberman Second Order Parabolic Differential Equations, World Scientific Pub. Co., Singapore, 1996.

[13] N.Nadirashvili, Nonuniqueness in the martingale problem and Dirichlet problem for uniformly elliptic operators, Ann. Scuola Norm. Sup. Pisa Cl.Sci.(4) 24 (1997), no.3, 537-549.

[14] C.Pucci Operatori ellittici estremanti, Ann. Mat.Pura Appl. (4) 72 (1966), 141-170.

[15] C.Pucci Limitazioni per soluzioni di equazioni ellittiche, Ann. Mat. Pura Appl. (4) 74 (1966) 15-30.

[16] M.V.Safonov, Harnack inequality for elliptic equations and Hölder property of their solutions, (Russian) Izv. Akad. Nauk SSSR Ser. Mat. 52 (1988), no. 6, 1272-1287, 1328; English translation in Math.USSR.Izv. 33, (1989) n.3, 597-612.

[17] M.V.Safonov, Classical solution of second-order nonlinear elliptic equations, (Russian) Zap. Nauĉn. Sem. Leningrad. Otdel. Mat. Inst. Steklov. (LOMI) 96 (1980), 272-287; English translation in J. Soviet Math. 21, (1983) n.5, 851-863.

[18] M.V.Safonov On a weak uniqueness for some elliptic equations, Comm. Partial Differential Equations 19 (1994), no. 5-6, 943-957.

[19] K. Tso, On an Alexsandrov-Bakel'man type maximum principle for second order parabolic equations, Comm. Partial Differential Equations 10 (1985), no. 5, 543-553.

[20] L.WANG, On the regularity of fully nolinear parabolic equations I, Comm. Pure Appl. Math. Comm. 45 (1992), no. 1, 27-76.

(M. C. Cerutti) Dipartimento di Matematica - Politecnico di Milano, Piazza Leonardo DA Vinci, 32, 20133 MiLANO-Italy

E-mail address: cristina.cerutti@polimi.it

(A. Piro Grimaldi) Dipartimento di Matematica dell'Università di Cagliari, Viale Merello, 94, 09100 CAGLiARI-Italy

E-mail address: grimaldi@unica.it 